\title{
AN UPPER BOUND FOR RAMSEY NUMBERS
}

\author{
BY JACK E. GRAVER AND JAMES YACKEL
}

Communicated by V. Klee, July 13, 1966

The finite form of Ramsey's Theorem [2], states that there exists a function $h(n)$ so that if a graph $G$ has at least $h(n)$ points, then either $G$ contains a complete subgraph on $n$ points, or a set of $n$ independent points (a set of points with no edges between any pair). We note that the graphs to be considered have no loops and each pair of points are joined by at most one edge.

Define $f(k, n)$ to be the least integer so that every graph with $f(k, n)$ points contains a complete subgraph on $k$ points or contains a set of $n$ independent points. Erdös and Szekeres [1], proved that

$$
f(k, n) \leqq\left(\begin{array}{c}
k+n-2 \\
k-1
\end{array}\right) .
$$

This upper bound can be improved as we show that

$$
f(k, n) \leqq\left(\prod_{i=3}^{k} C_{i}\right) \frac{n^{k-1}}{(k-1) !}+o\left(n^{k-1}\right)
$$

where $0<C_{i}<1$ for $i=3,4,5, \cdots$, and in particular

$$
f(3, n) \leqq\left(\frac{111+(33)^{1 / 2}}{128}\right) \frac{n^{2}}{2}+o\left(n^{2}\right) .
$$

Definition 1. A graph $G$ will be called a Ramsey $(k, n)$ graph if it has no complete subgraph on $k$ points and no set of $n$ independent points.

Note that a Ramsey $(k, n)$ graph is not required to have the maximum number, $f(k, n)-1$, of points.

Definition 2. The complement of a point of a graph $G$ is the subgraph of $G$ obtained by deleting from $G$ the given point, all points joined to this point by an edge and all edges incident to these points.

REMARK 1 . The complement of a point in a Ramsey $(k, n)$ graph must be a Ramsey $(k, n-1)$ graph. This is obvious since the point is independent of all points in its complement.

REMARK 2. The set of points joined to a given point in a Ramsey $(k, n)$ graph together with their edges must be a Ramsey $(k-1, n)$ graph.

Proof of (1). Let $G_{n}$ be a Ramsey $(k, n)$ graph on $N$ points. Since 
by Remark 2 each point has valence less than $f(k-1, n)$ we see that the number of edges of $G_{n}$ is bounded above by

$$
\frac{\{f(k-1, n)-1\} N}{2} \text {. }
$$

We now wish to get a lower bound on the number of edges of $G_{n}$ and we proceed as follows:

Let $P_{n}$ be a point of $G_{n}$ which has minimum valence in $G_{n}$. Denote the complement of $P_{n}$ by $G_{n-1}$. By Remark $1, G_{n-1}$ is a Ramsey $(k, n-1)$ graph. We now proceed inductively choosing $P_{i}$ a point of $G_{i}$ which has minimum valence in $G_{i}$ and letting $G_{i-1}$ denote the complement of $P_{i}$ taken with respect to the graph $G_{i}$. Also note that since $G_{i}$ is a Ramsey $(k, i)$ graph, $G_{i-1}$ will be a Ramsey $(k, i-1)$ graph. This sequence of points $\left\{P_{i}\right\}$ is obviously an independent set of points of $G_{n}$ hence there are at most $n-1$ points chosen by this process. In our notation we will proceed as though this sequence contained $n-1$ elements.

Let $v_{i}$ denote the valence of $P_{i}$ and note that since $G_{i}$ was a Ramsey $(k, i)$ graph, by Remark $2,0 \leqq v_{i} \leqq f(k-1, i)-1$. The number of edges of $G_{n}$ removed at the $i$ th step of this process can now be estimated. By Remark 2 we see that the points joined to $P_{i}$ have at most $f(k-2, i) f(k-1, i) / 2$ edges between them so that since each point has valence $v_{i}$ or more we have removed at least

$$
v_{i}^{2}-f(k-2, i) f(k-1, i) / 2 \text { edges of } G_{n} .
$$

This gives a lower bound on the number of edges of $G_{n}$ to be

$$
\sum_{i=2}^{n}\left\{v_{i}^{2}-\frac{f(k-2, i) f(k-1, i)}{2}\right\}
$$

On the other hand we note that the $i$ th step of the process removed exactly $v_{i}+1$ points of $G_{n}$ and (3) can be rewritten as

$$
\frac{f(k-1, n)}{2} \sum_{i=2}^{n}\left(v_{i}+1\right) \text {. }
$$

Combining this with (4) gives us

$$
\sum_{i=2}^{n}\left\{v_{i}^{2}-\frac{f(k-2, i) f(k-1, i)}{2}\right\} \leqq \frac{f(k-1, n)}{2} \sum_{i=2}^{n}\left(v_{i}+1\right) .
$$

Since we are interested only in the order of magnitude of these terms we will let 


$$
v_{i}=C i^{k-2}-e_{i}
$$

where $f(k-1, i) \leqq C i^{k-2}+o\left(i^{k-2}\right)$. Note that the bound of Erdös and Szekeres guarantees the existence of such a number $C$. We will show that

$$
\sum_{i=2}^{n} e_{i}=O\left(n^{k-1}\right)
$$

hence this will show the existence of the constant $C_{k}<1$.

With this change of notation in (5) and by combining all terms of lower order we assert

$$
\sum_{i=2}^{n}\left(C i^{k-2}-e_{i}\right)^{2} \leqq \frac{C n^{k-2}}{2} \sum_{i=2}^{n}\left(C i^{k-2}-e_{i}\right)+o\left(n^{2 k-3}\right) .
$$

Hence if $\sum e_{i}=o\left(n^{k-1}\right)$ we would have

$$
\sum_{i=2}^{n}\left(C i^{k-2}\right)^{2} \leqq \frac{C n^{k-2}}{2} \sum_{i=2}^{n}\left(C i^{k-2}\right)+o\left(n^{2 k-3}\right)
$$

or

$$
\frac{n^{2 k-3}}{2 k-3} \leqq \frac{n^{2 k-3}}{2 k-2}+o\left(n^{2 k-3}\right),
$$

which is clearly impossible. Hence by (6), $N=\sum C i^{k-2}-\sum e_{i}$. Therefore $N \leqq C\left(C_{k}\right) n^{k-1} / k-1+o\left(n^{k-1}\right)$ and (1) follows.

Remark 3. From (7) and for $k=3$, where we know $C=1$, we have

$$
\sum_{i=2}^{n}\left(i-e_{i}\right)^{2} \leqq \frac{n}{2} \sum_{i=2}^{n}\left(i-e_{i}\right)+o\left(n^{3}\right)
$$

which easily shows that

$$
C_{3} \leqq \frac{111+(33)^{1 / 2}}{128} .
$$

Remark 4. Estimation of the constant $C_{k}, k=3,4,5, \ldots$ shows that

$$
2\left(1-\frac{1}{k}\right)^{k-1} \leqq C_{k} \leqq\left(1-\frac{1}{k^{2}}\right)^{k-2} \frac{(k+2)(k-1)}{k^{2}} .
$$

\section{REFERENCES}

1. P. Erdös and G. Szekeres, On a combinatorial problem in geometry, Composition Math. 2 (1935), 463-470. 
2. F. P. Ramsey, On a problem of formal logic, Proc. London Math. Soc. (2) 30 (1930), 264-286.

Dartmouth College

\section{COUNTEREXAMPLE TO EULER'S CON JECTURE ON SUMS OF LIKE POWERS}

BY L. J. LANDER AND T. R. PARKIN

Communicated by J. D. Swift, June 27, 1966

A direct search on the CDC 6600 yielded

$$
27^{5}+84^{5}+110^{5}+133^{5}=144^{5}
$$

as the smallest instance in which four fifth powers sum to a fifth power. This is a counterexample to a conjecture by Euler [1] that at least $n n$th powers are required to sum to an $n$th power, $n>2$.

\section{REFERENCE}

1. L. E. Dickson, History of the theory of numbers, Vol. 2, Chelsea, New York, 1952, p. 648. 